\title{
Nachruf für Prof. Dr. Dieter Buck-Gramcko
}

\author{
In Memoriam Prof. Dr. Dieter Buck-Gramcko
}

Bibliografie

DOI http://dx.doi.org/

10.1055/s-0032-1329971

Handchir Mikrochir Plast Chir

2012; 44: 319-320

(c) Georg Thieme Verlag KG

Stuttgart · New York

ISSN 0722-1819

Korrespondenzadresse

Dr. Rolf Habenicht

Abt. für Handchirurgie

Kinderkrankenhaus Wilhelmstift

Liliencronstraße 130

22149 Hamburg

r.habenicht@kkh-wilhelmstift.de

\section{Ein Leben für die Handchirurgie \\ $\nabla$}

Unter dieses Motto hat Prof. Dr. Dieter BuckGramcko seine letzte Veröffentlichung von 100 Lebensbildern handchirurgischer Kollegen aus aller Welt gestellt, zu denen auch er gehörte, und dieses Motto charakterisiert sicher auch sein Leben.

Prof. Dieter Buck-Gramcko ist am 3. Oktober 2012 nur wenige Tage nach seiner Frau und kurz vor seinem 85. Geburtstag in Hamburg verstorben.

Dieter Buck-Gramcko wurde am 28. Oktober 1927 in Hamburg als Sohn des Orthopäden Paul Buck geboren. Nach Beendigung seiner Schulzeit, die durch seine Teilnahme am 2. Weltkrieg als Luftwaffenhelfer und später als Panzergrenadier sowie durch eine kurze Kriegsgefangenschaft unterbrochen wurde, studierte er von 1947 bis 1952 Medizin, zunächst in Hamburg und danach in Düsseldorf.

Schon während seiner Weiterbildung zum Chirurgen wurde sein Interesse an der Handchirurgie durch Oberarzt Alois Tietze im Unfallkrankenhaus Graz geweckt. Kurz nach seiner Rückkehr nach Hamburg in die chirurgische Abteilung des Allgemeinen Krankenhauses St. Georg unter der Leitung von Chefarzt Hans Wilhelm Buchholz wurde er beauftragt, die schwerwiegenden Handverletzungen zu versorgen. Ein 3-monatiger Aufenthalt bei Prof. Erik Moberg in Göteborg 1957 bestärkte ihn dann in seiner Überzeugung, sich der Handchirurgie vollends zu verschreiben. Dies war zudem der Beginn einer lang andauernden Freundschaft mit Erik Moberg.

1959 wurde Dieter Buck-Gramcko die Möglichkeit gegeben, das Berufsgenossenschaftliche Unfallkrankenhaus Hamburg-Boberg mit aufzubauen. 1963 wurde hier die erste eigenständige handchirurgische Abteilung in Deutschland unter seiner Leitung eröffnet. Die Arbeitsbedingungen waren seinen eigenen Aussagen nach so ideal, dass er bis zu seiner Pensionierung 1992 dort tätig war.

Hier gründete er auch einen deutschsprachigen handchirurgischen Literaturzirkel und führte 1960 das erste deutschsprachige handchirurgische Symposium durch. Dies war der Beginn der deutschsprachigen Arbeitsgemeinschaft für Handchirurgie und letztendlich auch der Deutschen Gesellschaft für Handchirurgie, deren erster Präsident er im Jahr 1991 wurde und zu deren Ehrenpräsident er im Jahr 1994 ernannt wurde. Er war zudem Gründungsmitglied nationaler und internationaler Fachgesellschaften, wie der Europäischen und der Internationalen Gesell-

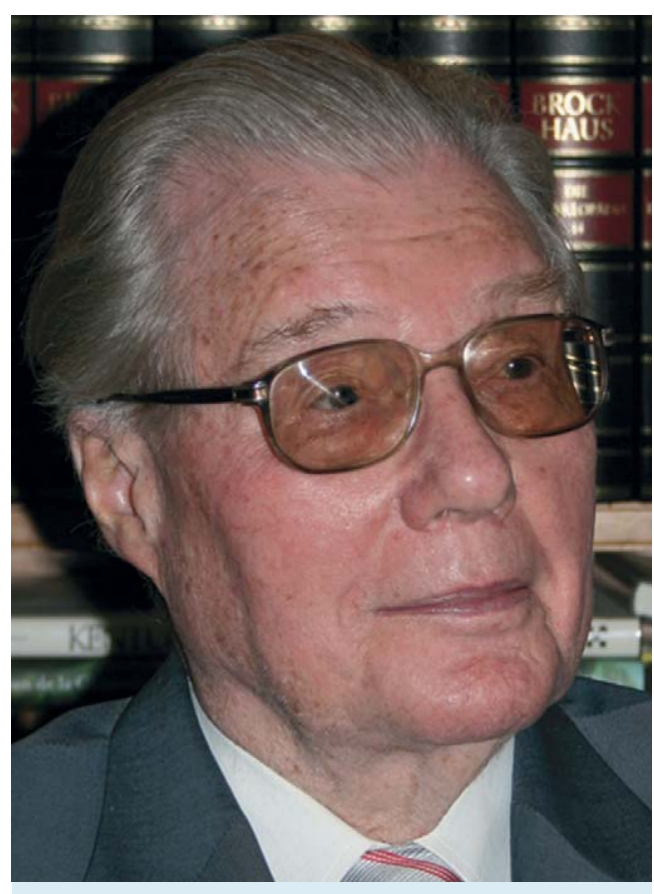

Prof. Dr. Dieter Buck-Gramcko

schaft für Handchirurgie und war Präsident der IFSSH von 1974 bis 1975.

Neben der allgemeinen Hand-, Mikro- und plastischen Chirurgie beschäftigte sich Dieter BuckGramcko seit Beginn der Contergan-(Talidomid-) Katastrophe in zunehmenden Maße mit Armfehlbildungen. Sein besonderes Interesse galt der hochgradigen Daumenhypoplasie, und er entwickelte seine Technik der Pollizisation, die heute noch weltweit eingesetzt wird. Mit der Pollizisation des Zeigefingers habilitierte er sich 1971. Sein Vortrag auf der Jahrestagung der Amerikanischen Gesellschaft für Handchirurgie zu diesem Thema brachte ihm die Ehrenmitgliedschaft der amerikanischen Gesellschaft ein, eine Ehrung, die nur wenigen Nicht-Amerikanern vergönnt ist. Diese Operation führte er auf seinen vielen Auslandsreisen weltweit durch. Bedingt durch seine vielen internationalen Kontakte und Freundschaften, wurde Hamburg zum „Mekka“ der Handchirurgie, das von vielen handchirurgischen Kollegen besucht wurde.

Neben seinen unzähligen Vorträgen umfasst seine wissenschaftliche Arbeit 168 Zeitschriftenartikel und 99 Buchkapitel sowohl als Herausgeber als auch als Autor. Er war Mitbegründer der deutschen Zeitschrift für Hand-, Mikro- und Plastische Chirurgie, deren Schriftleiter er für mehr als 30 Jahre war. Mit seinem letzten, 1998 
erschienenen und auf Englisch publizierten Buch „Congenital Malformations of the Hand and Forearm“, krönte er sein Lebenswerk.

Dieter Buck-Gramcko war Ehrenmitglied vieler nationaler und internationaler Fachgesellschaften. Unter seinen vielen Ehrungen erscheinen die Ernennung zum Honorary Fellow of the Royal College of Physicians and Surgeons in Glasgow 1980, die Ernennung zum Pioneer of Hand Surgery der IFSSH 1998 und die Verleihung des Bundesverdienstkreuzes 1992 besonders erwähnenswert.

Nach einer sehr schweren Erkrankung 2001 zog sich Dieter Buck-Gramcko weitgehend in das Privatleben zurück, zeigte aber nach wie vor reges Interesse an der weiteren Entwicklung der Handchirurgie.
Mit Dieter Buck-Gramcko verlieren wir einen Handchirurgen der ersten Generation, einen Freund und außergewöhnlichen Kollegen und den Mentor der deutschen Handchirurgie. Wir werden immer seiner gedenken und auch über seinen Tod hinaus von seiner handchirurgischen Arbeit profitieren.

Dr. Rolf Habenicht

Präsident

Deutsche Gesellschaft für Handchirurgie Hamburg, im Oktober 2012

Wir trauern um

\section{Herr Prof. Dr. med. Dieter Buck-Gramcko}

28.10.1927-3.10.2012

Als Mitbegründer und langjähriger Herausgeber der Handchirurgie, Mikrochirurgie, Plastischen Chirurgie hat er die Zeitschrift entscheidend geprägt. Seinem Engagement für die Zeitschrift gilt unsere tiefe Dankbarkeit. Wir werden sein Erbe stets in Erinnerung halten. Unser aufrichtiges Beileid gilt den Angehörigen, die innerhalb kürzester Zeit nicht nur ihn, sondern auch seine Ehefrau verloren haben.

Riccardo Giunta, Karl-Josef Prommersberger,

Georg Thieme Verlag 